Conclusions The mean number of painful procedures per NICU patient per day declined and analgesic treatment changed to a more tailored or individualized approach. Non-pharmacological pain- or stress reducing strategies like NIDCAP and sucrose were fully embedded in our pain management. As further reduction of the number of painful procedures is unlikely we should explore newer pharmacological agents and apply non-pharmacological interventions more frequently.

\section{PREDICTING THE EFFICACY OF ORAL SUCROSE IN REDUCING PAIN DURING OPHTALMOLOGICAL EXAMINATION FOR RETINOPATHY OF PREMATURITY: A PROSPECTIVE RANDOMISED STUDY}

doi:10.1136/archdischild-2012-302724.0190

${ }^{1} \mathrm{~N}$ Cullas Ilarslan, ${ }^{1} \mathrm{D}$ Dilli, ${ }^{2} \mathrm{EU}$ Kabataş, 'S Beken, 'B Aydın, 'A Zenciroğ lu, 'N Okumuş, 1Neonatology, Dr Sami Ulus Maternity, Childrens Education and Research Hospital, Division of Neonatology; ${ }^{2}$ Opthalmology, Dr Sami Ulus Maternity, Childrens Education and Research Hospital, Ankara, Turkey

Background and aim Retinopathy of prematurity (ROP) is one of the major morbidity among preterm infants. Although, local anesthetics reduce pain to some extent, eye examination still remains as a painful procedure. We aimed to evaluate the effect of oral sucrose combined with local anesthetics for pain relief during ophthalmological examination.

Method A total of forty patients under 32 weeks of gestational age were included in the study. Infants were randomly assigned to receive either oral sucrose solution (Group-1; $\mathrm{n}=21$ ) or sterile water (Group-2; $n=19$ ) combined with topical proparacaine hydrochloride two minutes before examination. Pacifier was used in all patients as non-nutritive sucking during the study. Pain score was evaluated by premature infant pain profile (PIPP) scale. Each infant was videorecorded during and after the procedure.

Results Both groups were similar in terms of gestational age, birth weight, postnatal age and actual weight. There was no significant difference between groups in behavioral state, heart rate and oxygen saturation before the examination. At speculum insertion, heart rate variability was similar in both groups whereas oxygen desaturation was apparent in Group-2 (Group-1: $1.7 \pm 0.8$ and Group-2: $2.5 \pm 0.6, \mathrm{p}=0.001)$ and PIPP scores were also lower in Group-1 (Group-1: $14.5 \pm 1.8$ and Group-2: 17.2 $\pm 1.7, \mathrm{p}=0.001$ ). Total time of crying was significantly shorter in Group-1 (Group-1: 58.8 \pm 12.1 and Group-2: 96.3 $\pm 24, \mathrm{p}=0.001$ )

Conclusion Procedural pain is known to have acute and even long term negative. behavioral and developmental effects in neonates. In our study, use of sucrose in addition to local anesthetics during ophthalmological examination is shown to attenuate pain.

\section{MORPHINE PREMEDICATION FOR INTUBATION IN PRETERM INFANTS - A PHARMACOKINETIC AND PHARMACOGENETIC REPORT}

doi:10.1136/archdischild-2012-302724.0191

${ }^{1} \mathrm{E}$ Norman, ${ }^{2} \mathrm{~L}$ Elens, ${ }^{3} \mathrm{M}$ Anderssson, ${ }^{3} \mathrm{O}$ Beck, ${ }^{2} \mathrm{R}$ van Shaik, ${ }^{3} \mathrm{~A}$ Rane, ${ }^{1,4 \mathrm{~V}}$ Fellman. 'Department of Pediatrics, Lund University and Skåne University Hospital Lund, Lund, Sweden; 'Department of Clinical Chemistry, Erasmus MC, Rotterdam, Netherlands Antilles; ${ }^{3}$ Division of Clinical Pharmacology, Lab Medicine, Karolinska Institute, Karolinska University Hospital, Stockholm, Sweden; ${ }^{4}$ Children's Hospital, University of Helsinki, Helsinki, Finland

Background and aims Morphine is used in preterm infants, but data are scars on pharmacokinetics (PK), pharmacodynamics (PD) and pharmacogenetics (PG). We aimed to study $\mathrm{PK}$ and PD/PG relation of morphine in infants included in a RCT comparing morphine with short-acting analgosedation as premedication for intubation.
Methods 17 preterm infants with a median (IOR) gestational age of 26.6 (25.1-28.7) w, birth weight 924 (721-1240) g and postnatal age $136(17.5-322)$ h were randomized to receive morphine $(0.3 \mathrm{mg} / \mathrm{kg})$.

Blood samples for morphine, M6G and M3G concentrations were collected before administration, 20 min, 6 and $24 \mathrm{~h}$ after intubation. DNA was isolated from salivary swabs to genotype 18 polymorphisms in 12 genes using Taqman assays. Pain assessment (ALPS-0 and EDIN scales) was performed and additional morphine boluses were offered accordingly. The morphine level/pain score relation and the genotype influence on time to achieve a low pain score was calculated.

Results In infants receiving no additional doses, clearance was $1.5-3.3 \mathrm{ml} / \mathrm{kg} / \mathrm{min}$ in 5 infants of $5-34 \mathrm{~h}$ and 9.9 in one infant of 332 $\mathrm{h}$ postnatal age.

Both morphine and morphine+M6G/5 correlated with mean ALPS-0 score at $6 \mathrm{~h}(\mathrm{p}=0.02$ and 0.04$)$ and $24 \mathrm{~h}(\mathrm{p}=0.01$ and 0.02$)$. The COMT rs4680G $>$ A (Val158Met) SNP was significantly correlated with time to reach the lowest pain score. COMT rs4680A patients experienced a faster response to opioids compared to rs4680GG patients in both groups ( $p=0.001$ and $p=0.072$ ).

Conclusions Morphine clearance is dependent on postnatal age in premature infants. Genotyping would improve individual dosing of opioids during NICU-care.

\section{ANALGESIA MODULATES CORTICAL RESPONSE TO PAINFUL PROCEDURES IN EXTREMELY PRETERM NEWBORN INFANTS}

doi:10.1136/archdischild-2012-302724.0192

M Bartocci, E Thorn, H Lagercrantz. Dept. of Neonatology, Karolinska University Hospital, Karolinska Institutet, Stockholm, Sweden

Introduction Proper pain management in the neonatal intensive care unit (NICU) is essential. The clinical pain assessment depends on objective measurement of indirect behavioural and physiological pain indicators. Cortical pain processing has been observed in preterm infants from 24 weeks gestational age but no study has focused exclusively on cortical pain response in extremely premature infants ( $\leq 28$ weeks gestational age).

Aims This study aimed to demonstrate cortical pain processing in extremely preterm infants. Furthermore, the study aimed to investigate the impact of analgesic drugs on cerebral haemodynamics and the relationship between behavioural and cortical pain responses.

Material and Methods A clinical study was performed in the NICU, including preterm infants $\leq 28$ weeks gestational age or with a birth weight $\leq 1500$ grams. Patients with severe on-going intraventricular haemorrhage or hydrocephalus were excluded. The infants were studied during routinely performed venepunctures and endotracheal tube suctions. Near-infrared spectroscopy was used for the study of cortical activity, parallel to observation of systemic haemodynamics and pain assessment with the Premature Infant Pain Profile and Échelle Douleur Inconfort Nouveau-Né.

Results During the procedures significant increases in the cerebral concentration of oxygenated haemoglobin were observed bilaterally during venepuncture and unilaterally during endotracheal tube suctions. Simultaneously, minor alterations in systemic haemodynamics occurred. The cerebral pain response was significantly reduced by analgesia.

Conclusions These results indicate the existence of cortical pain processing in very premature infants. Analgesia reduces this cortical response. CORTICAL MORPHOLOGY AND CONNECTIVITY

doi:10.1136/archdischild-2012-302724.0193 
'J Dubois, ' $\mathrm{G}$ Dehaene-Lambertz, ${ }^{2} \mathrm{JF}$ Mangin, ${ }^{3} \mathrm{D}$ Le Bihan, ${ }^{4} \mathrm{PS}$ Hüppi, ${ }^{5} \mathrm{~L}$ Hertz-Pannier. 'Cognitive Neuroimaging Unit, U 992, INSERM; ' LNAO; 'NeuroSpin, CEA, Gif-surYvette, France; ${ }^{4}$ Pediatrics Department, Geneva University Children's Hospital, Geneva, Switzerland; 5 LBIOM, CEA, Gif-sur-Yvette, France

Studying how the brain develops and becomes functional is a prerequisite to understand the complexity of developmental pathologies. Thanks to Magnetic Resonance Imaging (MRI), it is now possible to image the baby's brain non-invasively, and to provide subtle correlations between its anatomical development and the early acquisition of cognitive functions.

But imaging the immature brain implies several constraints at the levels of data acquisition and post-processing, because the baby may move, the examination length must then remain short, and because the size of cerebral structures and the image contrast are very different in comparison with the adult brain. Dedicated methodologies must then be applied to provide valid and instructive information on the development of cortical and white matter networks.

In this presentation, I will describe two recent MRI studies which evaluated the setting up of cortical morphology and connectivity under an original perspective. The first study has focused on the cortical folding patterns (formation of gyri and sulci), in preterm newborns from six to eight months of gestational age, by using T2-weighted conventional MRI. The second study has mapped the organization and maturation of major white matter fiber bundles (commissural, projection, association and limbic fascicles), in one- to four-months-old healthy infants, with diffusion tensor imaging (DTI) and tractography.

Both MRI studies have highlighted spatio-temporal differences in the maturation of brain regions, as well as early anatomical asymmetries between cerebral hemispheres. They have also emphasized the relationships between the structural development of the brain and the infant's psycho-motor acquisitions after birth.

\section{ARTERIAL SPIN LABELING MAGNETIC RESONANCE IMAGING TO ASSESS NEONATAL BRAIN PERFUSION}

doi:10.1136/archdischild-2012-302724.0194

${ }^{1} \mathrm{JB}$ De Vis, ${ }^{2} \mathrm{KJ}$ Kersbergen, ${ }^{1} \mathrm{ET}$ Petersen, ${ }^{1} \mathrm{~J}$ Hendrikse, ${ }^{2} \mathrm{~T}$ Alderliesten, ${ }^{2} \mathrm{~F}$ Groenendaal, ${ }^{2}$ LS de Vries, ${ }^{2}$ MJNL Benders. ${ }^{1}$ Radiology, UMC Utrecht; ${ }^{2}$ Neonatology, Wilhelmina Children's Hospital/UMC, Utrecht, The Netherlands

Background and aim Little is known about brain perfusion in neonates and its relation with brain development. The purpose of this study was to evaluate if Arterial Spin Labeling (ASL) Magnetic Resonance (MR) imaging can be used to evaluate the relation between brain perfusion and brain development.

Methods Pulsed ASL-images (Philips-3T) were acquired of 31 infants. Six infants were imaged at preterm age, 23 infants at term equivalent age (TEA) and 2 infants at 3 -months equivalent age $(3 \mathrm{~m})$. Serial MR imaging was performed in 4 infants. Total brain perfusion (TBP) was measured for each infant. Regions of interest (ROIs) were drawn within the occipital cortex (OC), the frontal cortex (FC) and one ROI covered the basal ganglia and thalamus (BGT). Perfusion values measured in the ROIs were expressed relative toTBP.

Results TBP increased from preterm age (7.1 $\mathrm{ml} / 100 \mathrm{~g}$. $\mathrm{min})$ to TEA $(12.6 \mathrm{ml} / 100 \mathrm{~g} \cdot \mathrm{min})$ to $3 \mathrm{~m}(30.2 \mathrm{ml} / 100 \mathrm{~g} \cdot \mathrm{min})$. A relative decrease in perfusion towards the BGT and a relative increase towards the $\mathrm{OC}$ and $\mathrm{FC}$ was measured with increasing postconceptional age [fig 1]. Serial ASL images of one infant scanned at preterm [Fig.2a] and TEA [Fig.2B] are shown.

Conclusions Arterial Spin Labeling MR images reflect the anatomical and functional maturation of the brain in neonates and are in agreement with previously obtained PET-images.

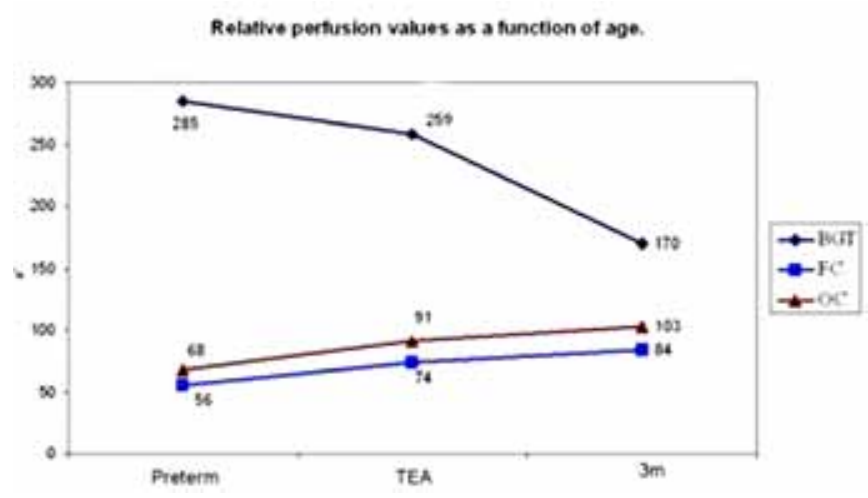

Abstract 194 Figure 1

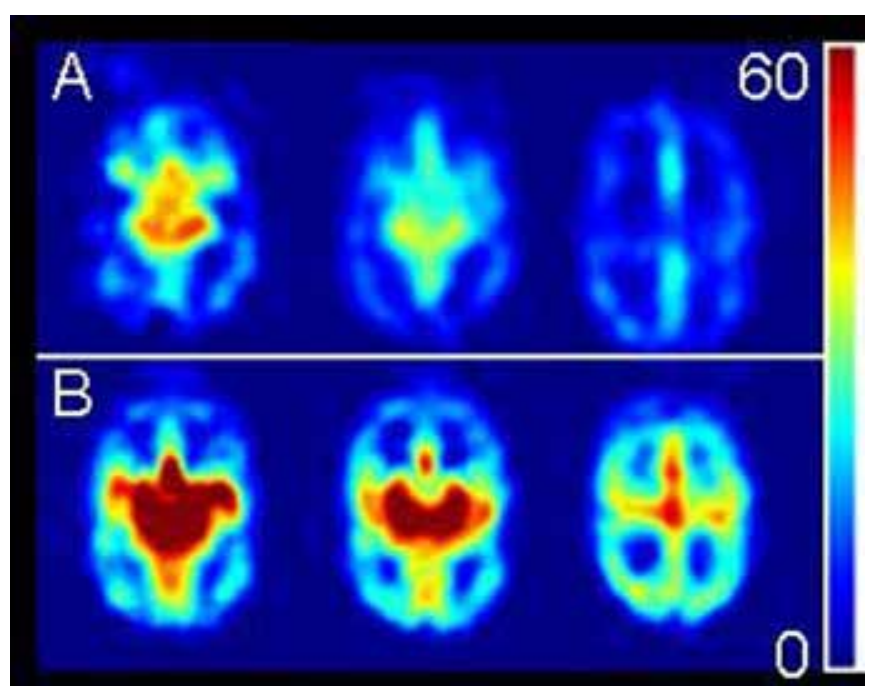

Abstract 194 Figure 2

\section{REGIONAL CHANGES OF CORTICAL THICKNESS AT 6 YEARS OF AGE IN PRETERM BORN CHILDREN}

doi:10.1136/archdischild-2012-302724.0195

'L Vasung, ${ }^{2} E$ Fischi Gomez, ${ }^{3} \mathrm{M}$ Monnier, ${ }^{4} \mathrm{AC}$ Evans, ${ }^{2} \mathrm{~F}$ Lazeyras, ${ }^{1} \mathrm{C}$ Borradori Tolsa 'PS Hüppi. 'Pediatrics, Service de Développement et Croissance, Geneva University Children's Hospital; ${ }^{2}$ Radiology, Center for Biomedical Imaging, Geneva University Hospital, Geneva; ${ }^{3}$ Pediatrics, Children's Hospital, Lausanne, Switzerland; ${ }^{4}$ ACE, Clinical Trials Imaging Lab, McConnell Brain Imaging Centre, Montreal Neurological Institute, McGill University, Montréal, OC, Canada

Background The growing incidence of prematurely born children and the improvement of survival rates have been associated with highly problematic long term neurodevelopmental outcomes. Brain structural alterations associated with these mainly cognitive difficulties most likely involve cortical organization. This study presents new ways of assessing structural organization of the cortex through thickness measurements.

Subjects/methods Preterm infants (N=42, GA $28.7 \pm 3.1 \mathrm{wks})$ were scanned at 6 years of age using the 3T MRI scanner. High-resolution 3D T1 MRI images were analyzed using MNI tools (http://www. bic.mni.mcgill.ca/ alan/lab.html). IUGR preterm subjects $(\mathrm{N}=14)$ were identified by abnormal antenatal Doppler measurements and mean birth weights below $10^{\text {th }}$ percentile. Cortical thickness was computed between extracted cortical surfaces and analyzed using the SurfStat tools (http://www.math.mcgill.ca/keith/surfstat/).

Results GA at birth within all subjects showed positive correlation with cortical thickness measurements (bilateral precuneus, right 